\title{
The Apache Point Observatory Galactic Evolution Experiment (APOGEE) in Sloan Digital Sky Survey III (SDSS-III)
}

\author{
Steven R. Majewski ${ }^{1}$, John C. Wilson ${ }^{1}$, Fred Hearty ${ }^{1}$, \\ Ricardo R. Schiavon ${ }^{2}$, and Michael F. Skrutskie ${ }^{1}$ \\ ${ }^{1}$ Dept. of Astronomy, University of Virginia, Charlottesville, VA 22904-4325, USA \\ ${ }^{2}$ Gemini Observatory, 670 N. A’Ohoku Place, Hilo, HI 96720, USA
}

\begin{abstract}
The Apache Point Observatory Galactic Evolution Experiment (APOGEE) is a largescale, near-infrared ( $H$-band), high-resolution $(R \sim 30,000)$, high $S / N(\gtrsim 100)$ spectroscopic survey of Milky Way stellar populations. APOGEE will operate from $1.51-1.68 \mu \mathrm{m}$, a region that includes useful absorption lines from at least fifteen chemical species including $\alpha$, odd- $Z$, and iron peak elements. The APOGEE instrument has a novel design featuring 300 science fibers feeding light to a mosaiced VPH grating and a six-element camera encased in a liquid nitrogen-cooled cryostat. A three year bright-time observing campaign will enable APOGEE to observe approximately 100,000 red giants across the Galactic bulge, disk and halo.
\end{abstract}

Keywords. galaxies: interactions, Galaxy: halo, Galaxy: structure, stars: abundances

\section{The APOGEE Survey}

One of the four experiments in the Sloan Digital Sky Survey III (SDSS-III) suite is the Apache Point Observatory Galactic Evolution Experiment (APOGEE), a largescale, near-infrared, high-resolution $(R \sim 30,000)$ spectroscopic survey of Milky Way stellar populations. APOGEE will observe in the $H$ band, where extinction by dust is significantly less than at optical wavelengths (e.g., $A_{H} / A_{V}=0.16$ ); thus APOGEE will be the first survey that can effectively explore the dust-obscured, low-latitude Galaxy and thereby provide a vast, uniform database of chemical abundances and radial velocities for stars across all Galactic stellar populations (bulge, thin and thick disks, and halo). The survey will be conducted in a contiguous spectral region of $1.51 \mu \mathrm{m}$ to $1.68 \mu \mathrm{m}$ using a dedicated, 300-fiber, cryogenic, spectrograph being built at the University of Virginia and that will be coupled to the wide-field, Sloan 2.5-m telescope at Apache Point Observatory. APOGEE will use a significant fraction of the SDSS-III bright time during 2011-2014 to observe, at high signal-to-noise $(S / N)$, about 100,000 giant stars selected from the Two Micron All-Sky Survey (2MASS) down to a typical flux limit of $H=12-14$. With its high resolution and a limit of $S / N>100$, APOGEE will determine for its vast sample of stars both precision radial velocities (to better than $0.5 \mathrm{~km} \mathrm{~s}^{-1}$ external accuracy) and accurate abundance measurements spanning numerous chemical species, including for most stars: the most abundant metals in the universe - i.e. C, N, O; other $\alpha$-elements, including $\mathrm{Mg}, \mathrm{Si}, \mathrm{Ca}$, Ti and possibly $\mathrm{S}$; the odd- $Z$ elements $\mathrm{Al}, \mathrm{K}$ and possibly $\mathrm{Na}$; iron peak elements, including $\mathrm{Mn}, \mathrm{Fe}, \mathrm{Ni}$ and possibly $\mathrm{V}, \mathrm{Cr}$, and $\mathrm{Co}$; and perhaps a small number of neutron-capture species. With this large catalog of chemical abundance data for stars all across the Milky Way, the APOGEE survey will be able to address a number of key problems, including: (1) measuring in a relatively unbiased, uniform manner and with statistically large samples of stars the metallicity distributions and 


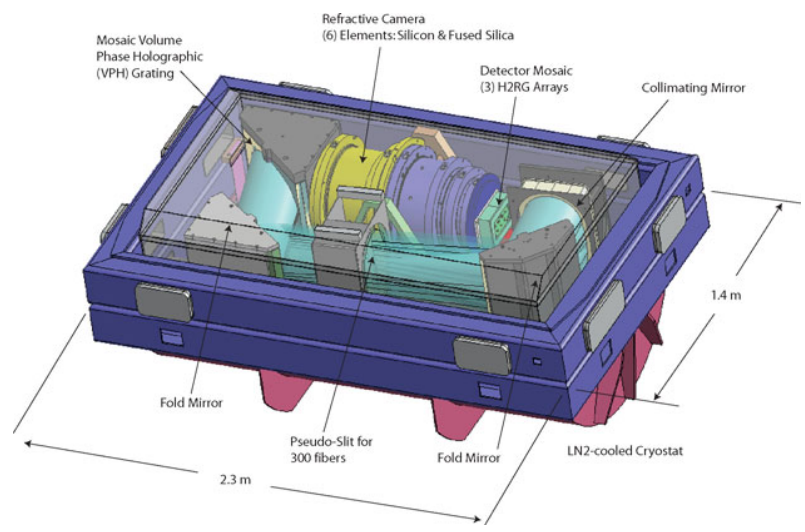

Figure 1. Solid model of the $2.3 \times 1.3-\mathrm{m}$ APOGEE cryostat showing the primary features of the spectrograph optical train. The cryostat, housed remotely from the telescope, is linked to the telescope focal plane by an $\sim 40$-m fiber optic train.

abundance patterns for numerous chemical species across the different Galactic stellar populations; (2) deriving spatial variations of these chemical distributions within these populations; (3) studying the processes of star formation, feedback, chemical mixing and chemical evolution in the Milky Way with sensitivity to numerous nucleosynthetic pathways; (4) constraining the evolution of the initial mass function, by inference from the differences in chemical yields produced by stars of different masses; and (5) using these extensive chemical data - combined with dynamical information provided by APOGEE radial velocities, proper motions from other surveys, as well as Galactic chemical evolution models - to unravel the overall formation and evolution of the Milky Way.

\section{The APOGEE Instrument}

The APOGEE instrument will be housed in a separate building at APO and be linked to the Sloan 2.5-m telescope via 40-m long fiber optic cables. A custom-made fiber optic coupler will be used to mate the long fiber run to the Sloan fiber plugplate cartridges. The standard Sloan cartridges are being modified to hold separate sets of fibers feeding different SDSS-III experiments that will be observing simultaneously: APOGEE (300 fibers), MARVELS (200 fibers), and, potentially, SEGUE-Bright (500 fibers). The APOGEE spectrograph features a compact, folded design to enable it to be encased in a liquidnitrogen-cooled cryostat with fiber feedthroughs bringing the 300 science fibers to a curved pseudo-slit (Fig. 1). The dispersing element is a volume phase holographic (VPH) grating, but the large collimated beam is larger than any presently-made commercial VPH; thus we are planning to use a VPH mosaic of size $265 \times 450$-mm made by Kaiser Optics. The $\sim 395$-mm optical diameter, six element spectrograph camera, built by New England Optical Systems, includes both silicon and fused silica lenses, only one of which is an asphere. To record spectra from $1.51 \mu \mathrm{m}$ to $1.68 \mu \mathrm{m}$ at the nominal $R \sim 30,000$ we use three Hawaii 2 RG arrays. This configuration is slightly undersampled in the dispersion direction on the blue end; to recover proper sampling the detector array assembly is held on a flex-pivot stage that enables precise lateral shifts in detector position. It is anticipated that all observations will be taken in pairs with half-pixel shifts. 